\title{
Histopathologic Changes in the Substantia Nigra Resulted by Paraquat Intoxication in Rat
}

\author{
Atefeh Sabbagh ${ }^{1}$, Abbas Tavasoly ${ }^{*}$, Jamileh Salar Amoli ${ }^{2}$, Seyed Hossein Mardjanmehr ${ }^{1}$ \\ ${ }^{1}$ Department of Pathology, Faculty of Veterinary Medicine, University of Tehran, Tehran, Iran \\ ${ }^{2}$ Department of Basic Sciences, Faculty of Veterinary Medicine, University of Tehran, Tehran, Iran
}

\section{ABSTRACT}

Introduction: Widespread agricultural use as well as increasing number of suicidal or accidental ingestion of paraquat in the last few years enhance the necessity to evaluate the mechanism of action and the complications, especially neuronal side-effects, of this herbicide. The aim of this study was to evaluate the histopathological alterations of the substantia nigra after paraquat intoxication in rats. Materials and Methods: 28 adult Wistar rats were randomly divided into three experimental and one control groups. Paraquat at different doses $(10,15$ and $20 \mathrm{mg} / \mathrm{kg}$ ) were injected intraperitoneally once a week for three consecutive weeks to experimental groups; normal saline was injected to control group. After 28 days, the brains were removed and sections from the compact part of the substantia nigra were stained by toluidine blue. Results: A significant enhancement of the number of dark neurons in the substantia nigra was observed in rats receiving 15 and $25 \mathrm{mg} / \mathrm{kg}$ of paraquat compared to control group. Conclusion: Our findings point to the harmful effects of paraquat on neurons of the substantia nigra. This suggests paraquat toxicity as a risk factor for neurological disorders, such as Parkinson's disease.

\section{Key words:}

1. Paraquat

2. Substantia Nigra

3. Pathology

*Corresponding Author: Abbas Tavasoly

E-mail:atavasoli@ut.ac.ir

doi: 10.18869/acadpub.shefa.4.4.41 


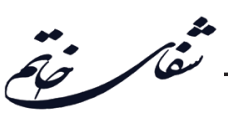

\section{تغييرات هيستو ياتولوزيك جسم سياه در نتيجةٌ مسموميت ياراكوات در موش صحرايى}

عاطفه صباغ'، عباس توسلى ا":، جميله سالار آملى'، سيد حسين مرجانمهر'

آخروه ياتولوزى، دانشكده داميزشكى، دانشكاه تهران، تهران، ايران

rكروه علوم بايه، دانشكده داميزشكى، دانشكاه تهران، تهران، ايران

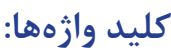

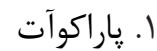

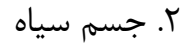

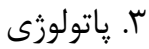

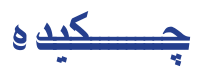

مقدمه: استفاده كشاورزى وسيع بهعلاؤ افزايش تعداد مصرف اتفاقى يا به قصد خودكشى از يارئ إكوات

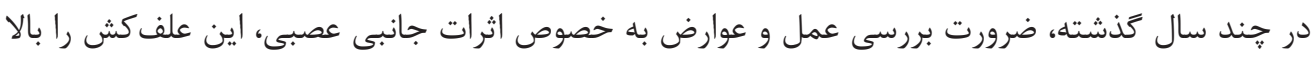

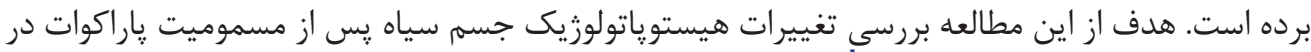

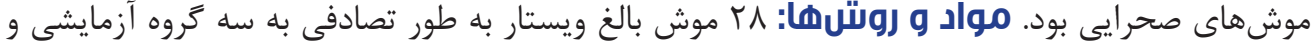

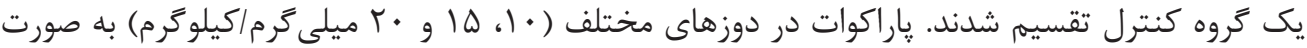

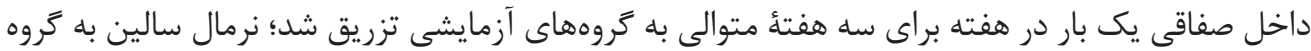

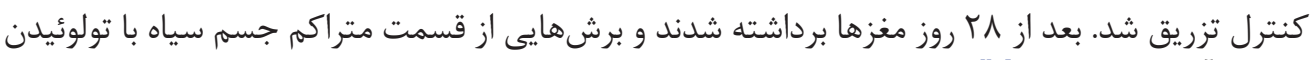

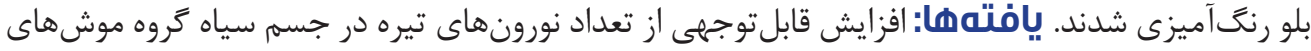

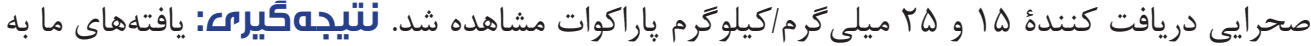

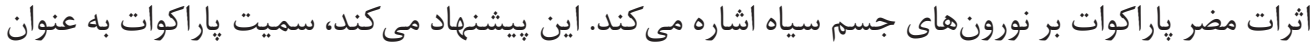

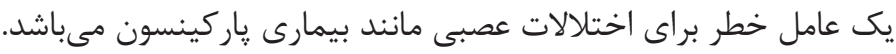




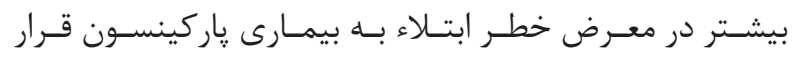

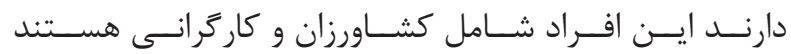

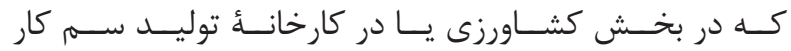

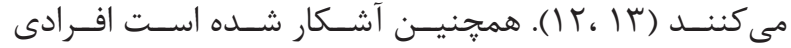

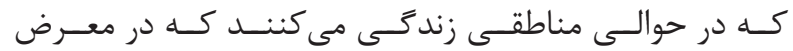

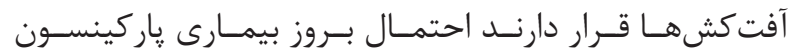

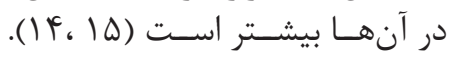

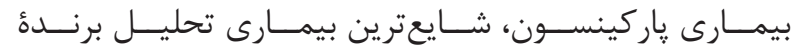

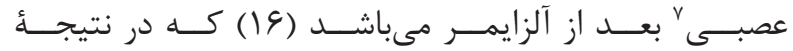

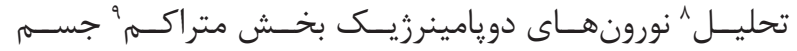

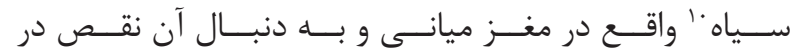

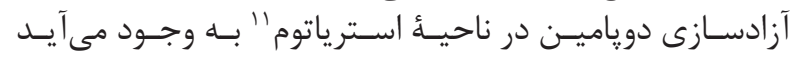

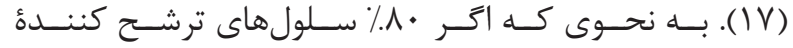

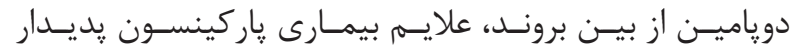

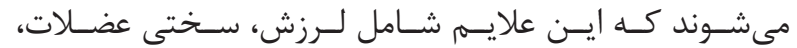

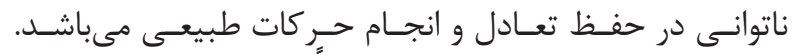

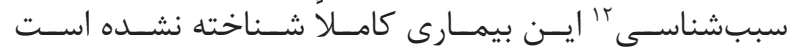

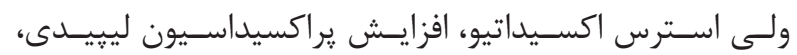

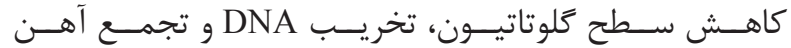

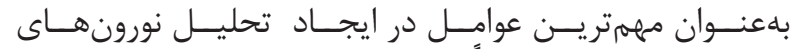

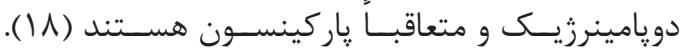

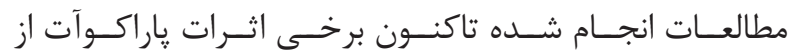

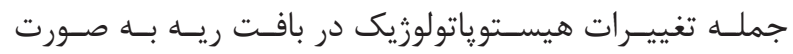

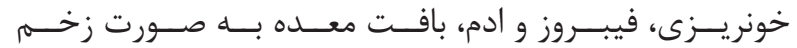

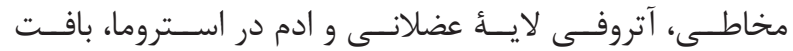

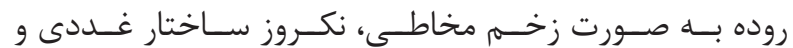

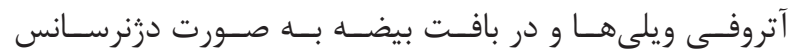

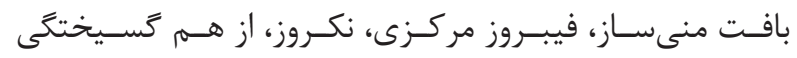

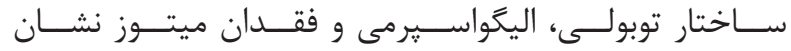

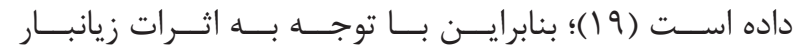

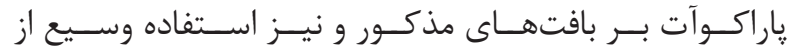

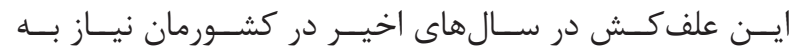

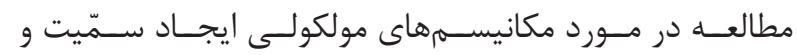

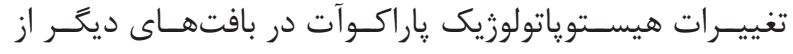

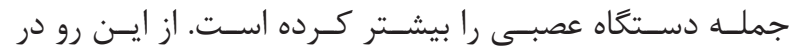

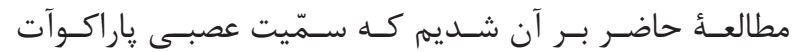

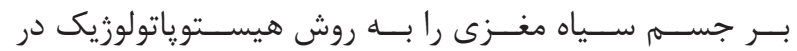
مـوش صحرايسى بررسـى كنيـهم. مواد و روشها حيوانات آزمايشخاهى

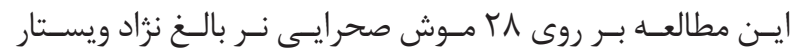

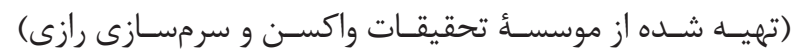

${ }^{1}$ Herbicides

${ }^{2}$ Bipyridylium

${ }^{3}$ Paraquat

${ }^{4}$ Diquat

${ }^{5}$ Morfam quat

${ }^{6}$ 1-methyl-4-phenyl-1,2,3,6-tetrahydropyridine

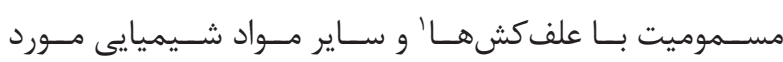

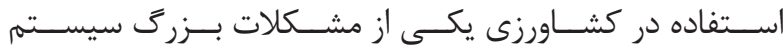

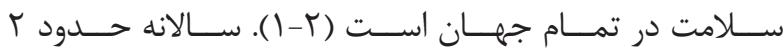

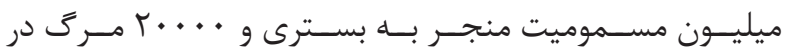

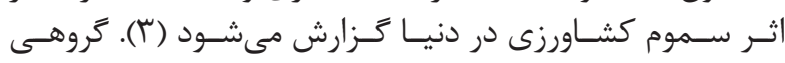

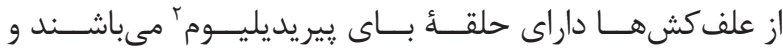

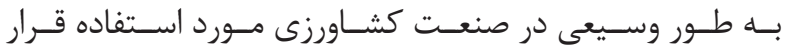

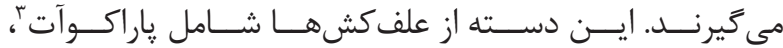

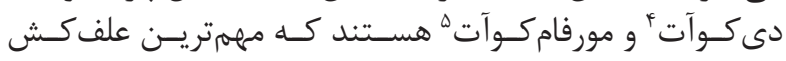

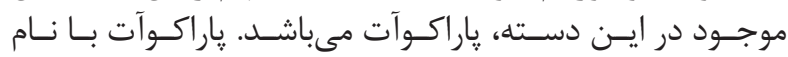

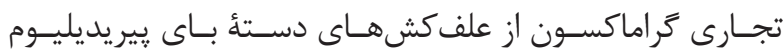

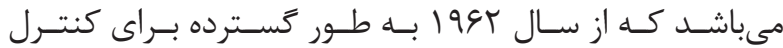

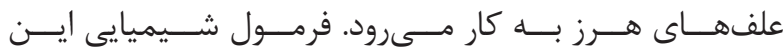

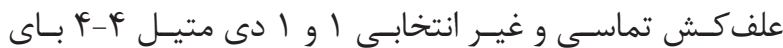

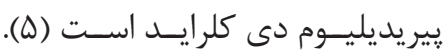

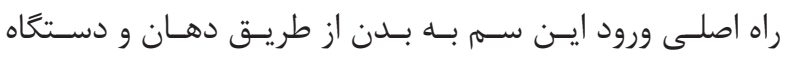

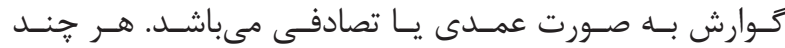

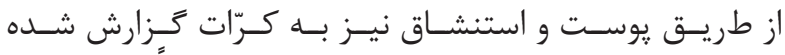

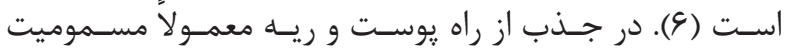

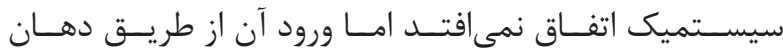

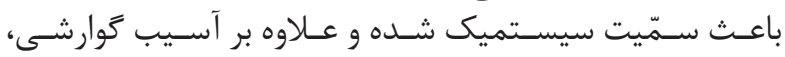

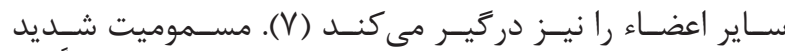

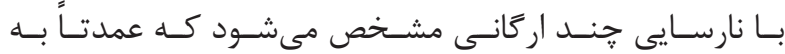

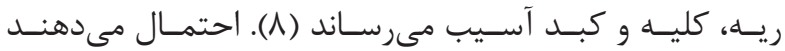

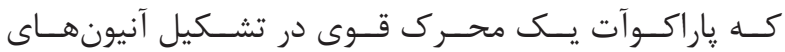

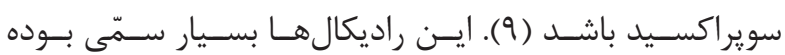

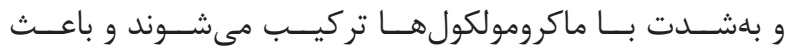

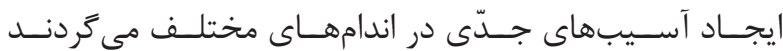

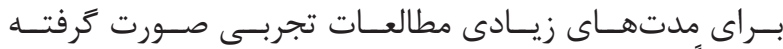

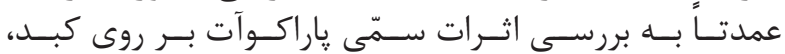

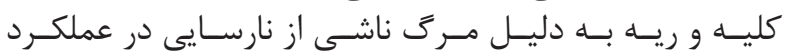

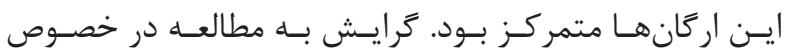

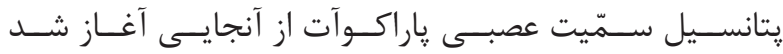

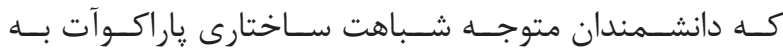

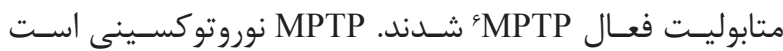

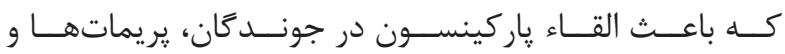

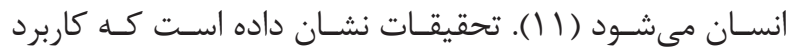

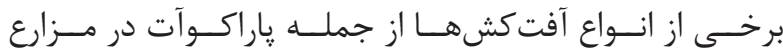

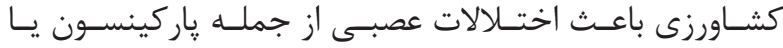

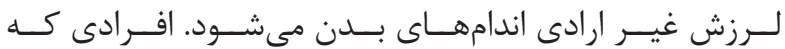

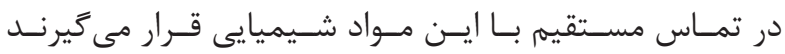

\footnotetext{
${ }^{7}$ Neurodegenerative

${ }^{8}$ Degeneration

${ }^{9}$ Compact part

${ }^{10}$ Substantia nigra

${ }^{11}$ Striatum

${ }^{12}$ Etiology
} 


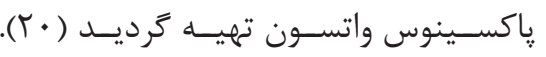

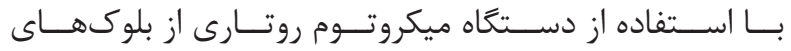

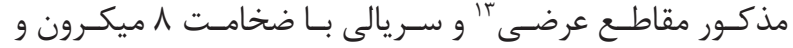

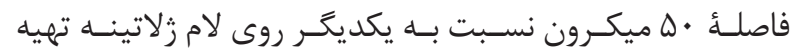

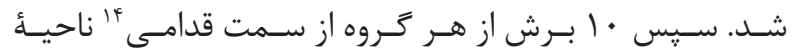

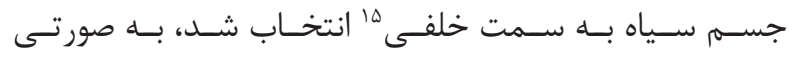

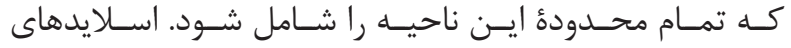

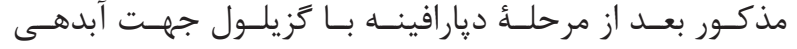

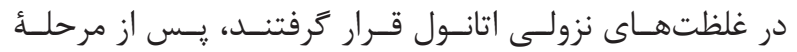

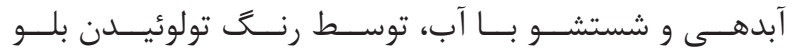

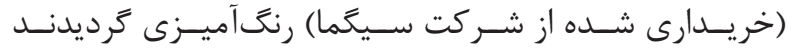

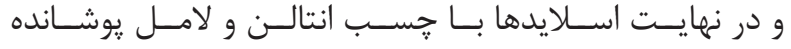

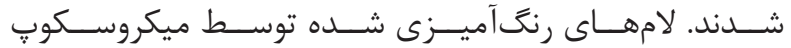

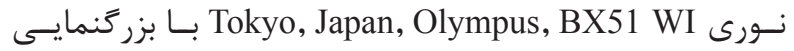

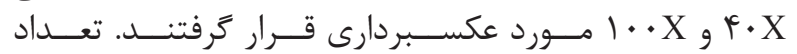

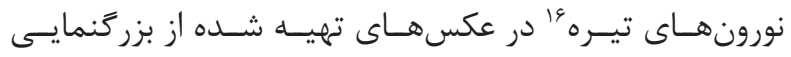

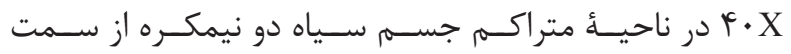

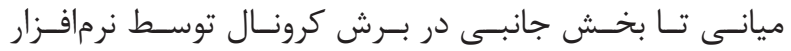

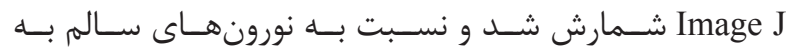

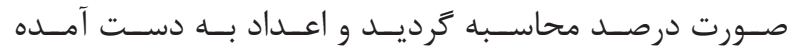

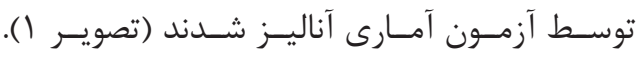

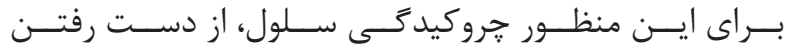

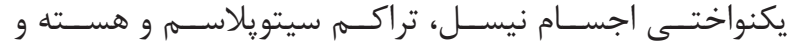

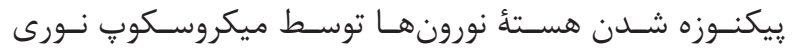

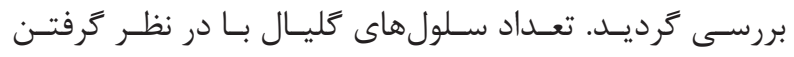

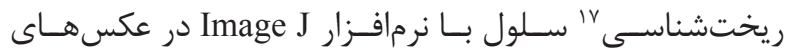

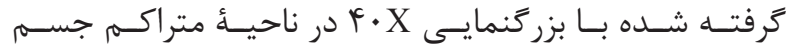

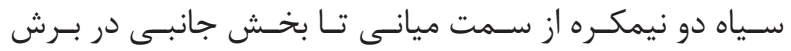

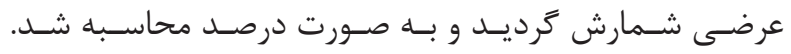

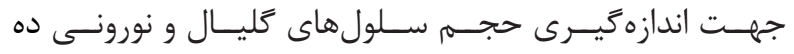

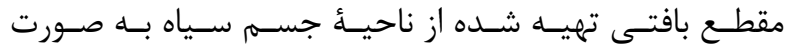

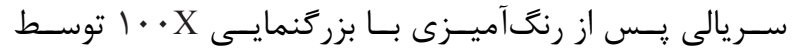

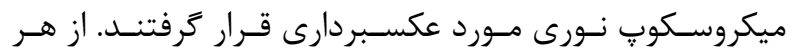

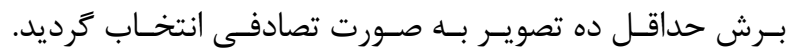

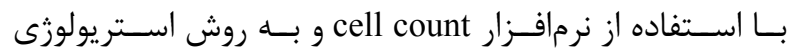

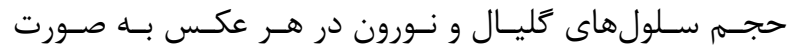

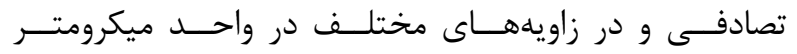

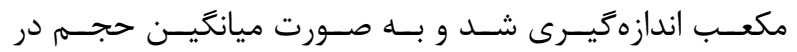

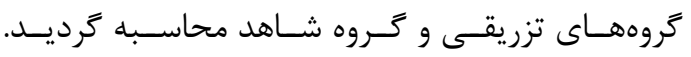

$$
\text { تجزيه و تحليل آمارى }
$$

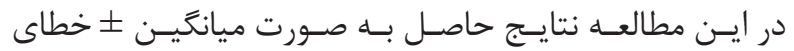

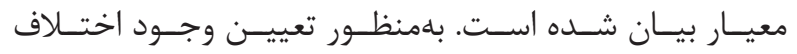

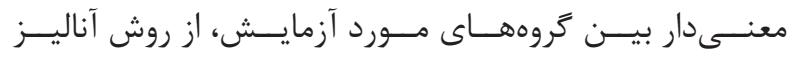

\section{${ }^{13}$ Coronal}

${ }^{14}$ Rostral

${ }^{15}$ Caudal

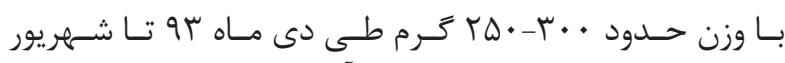

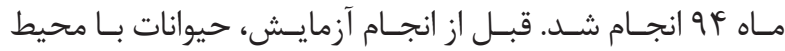

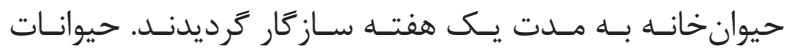

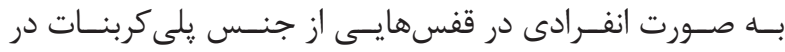

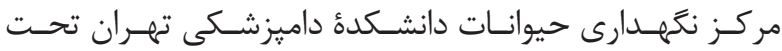

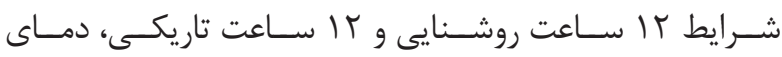

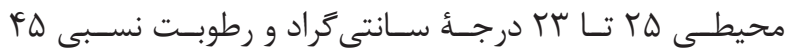

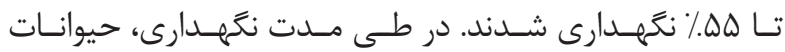

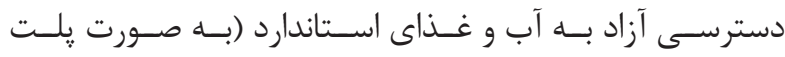

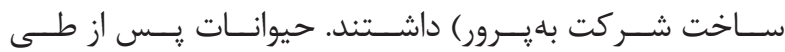

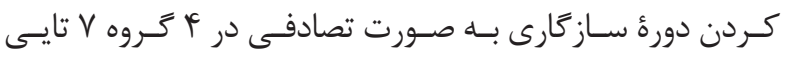

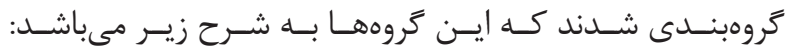

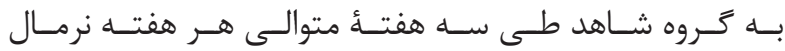

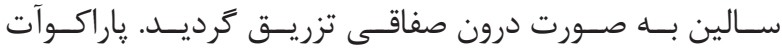

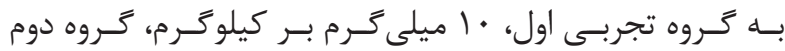

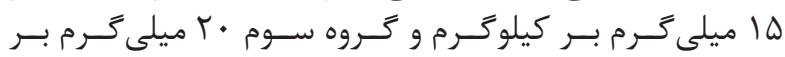

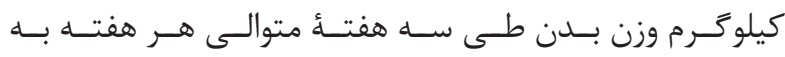

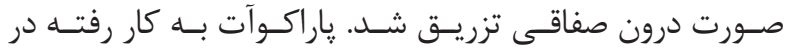

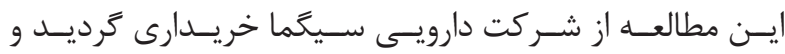

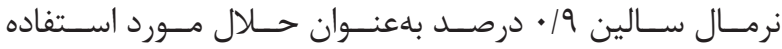

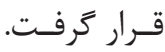

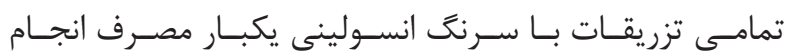

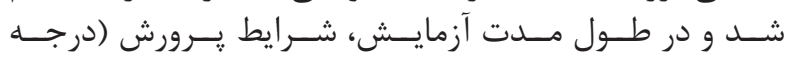

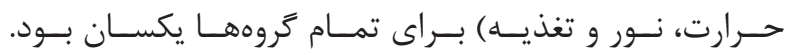

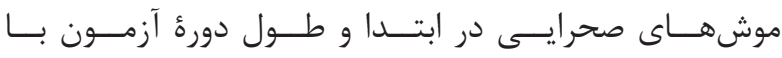

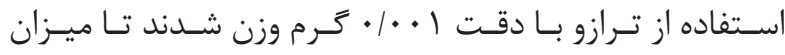

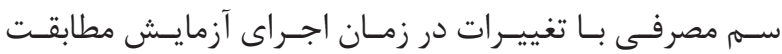

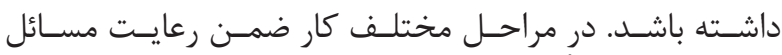

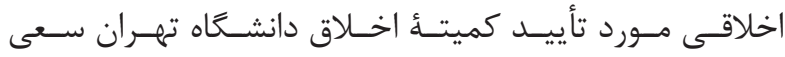

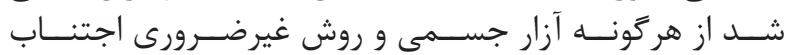

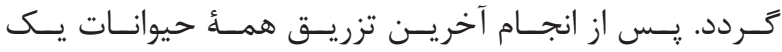

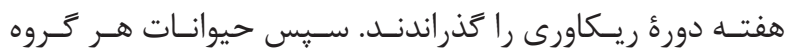

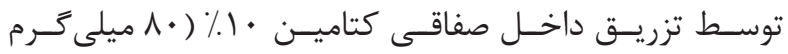

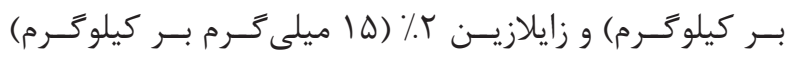

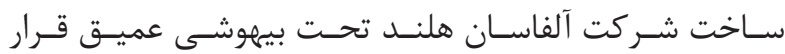

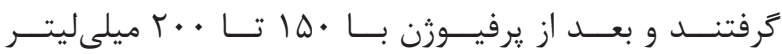

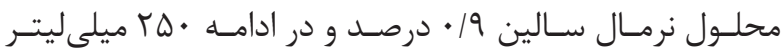

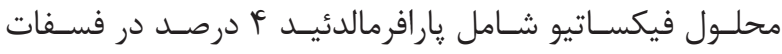

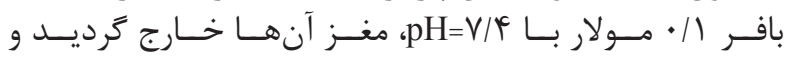

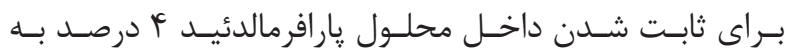

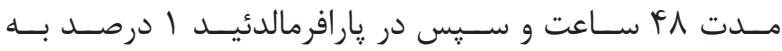

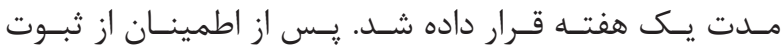

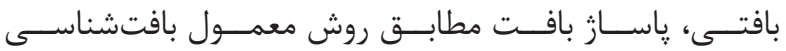

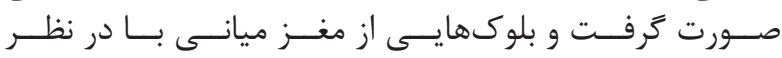

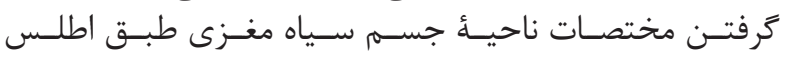

\footnotetext{
${ }^{16}$ Dark neurons

${ }^{17}$ Morphology
} 


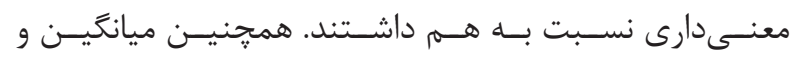

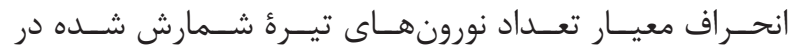

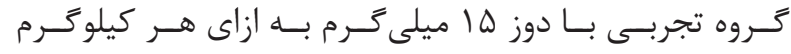

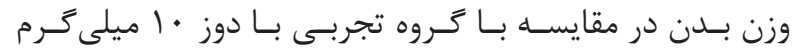

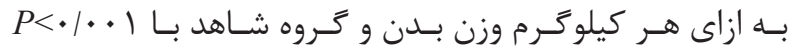

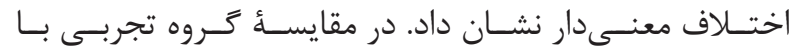

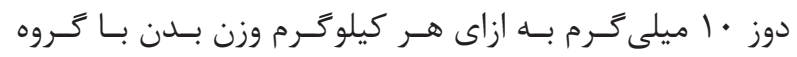

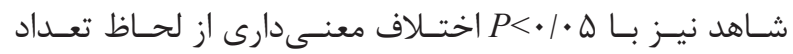

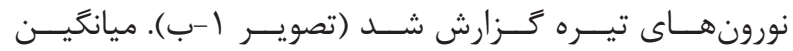

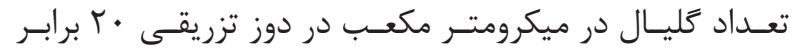

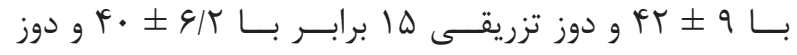

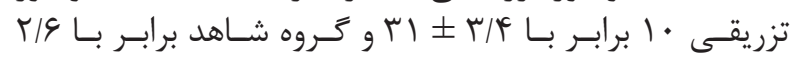

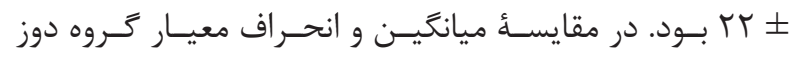

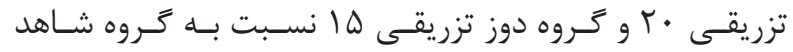

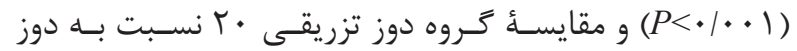

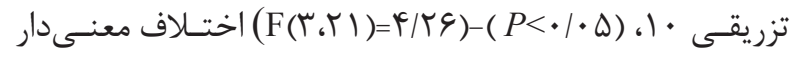

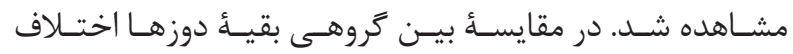

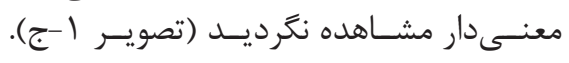

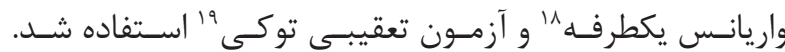

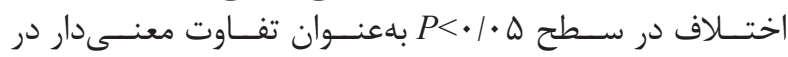

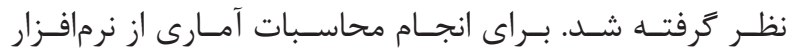
IBM SPSS Statistics

بافتهها

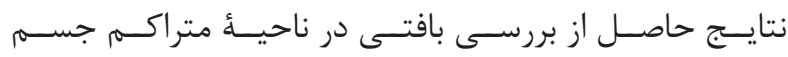

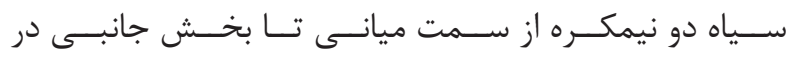

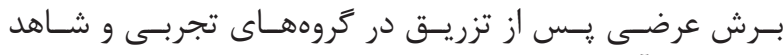

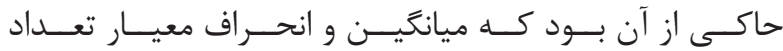

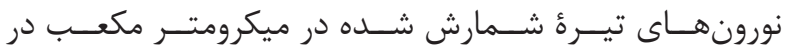

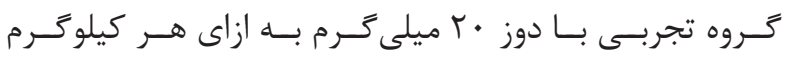

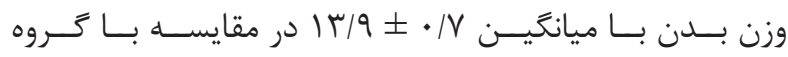

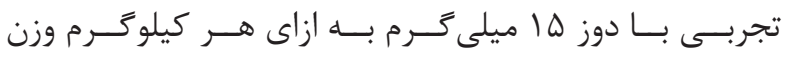

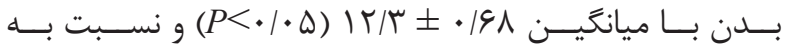

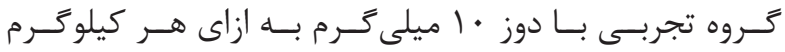

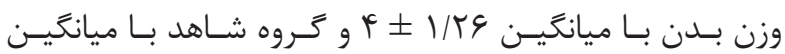

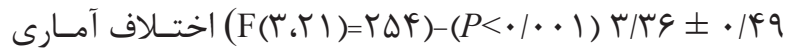

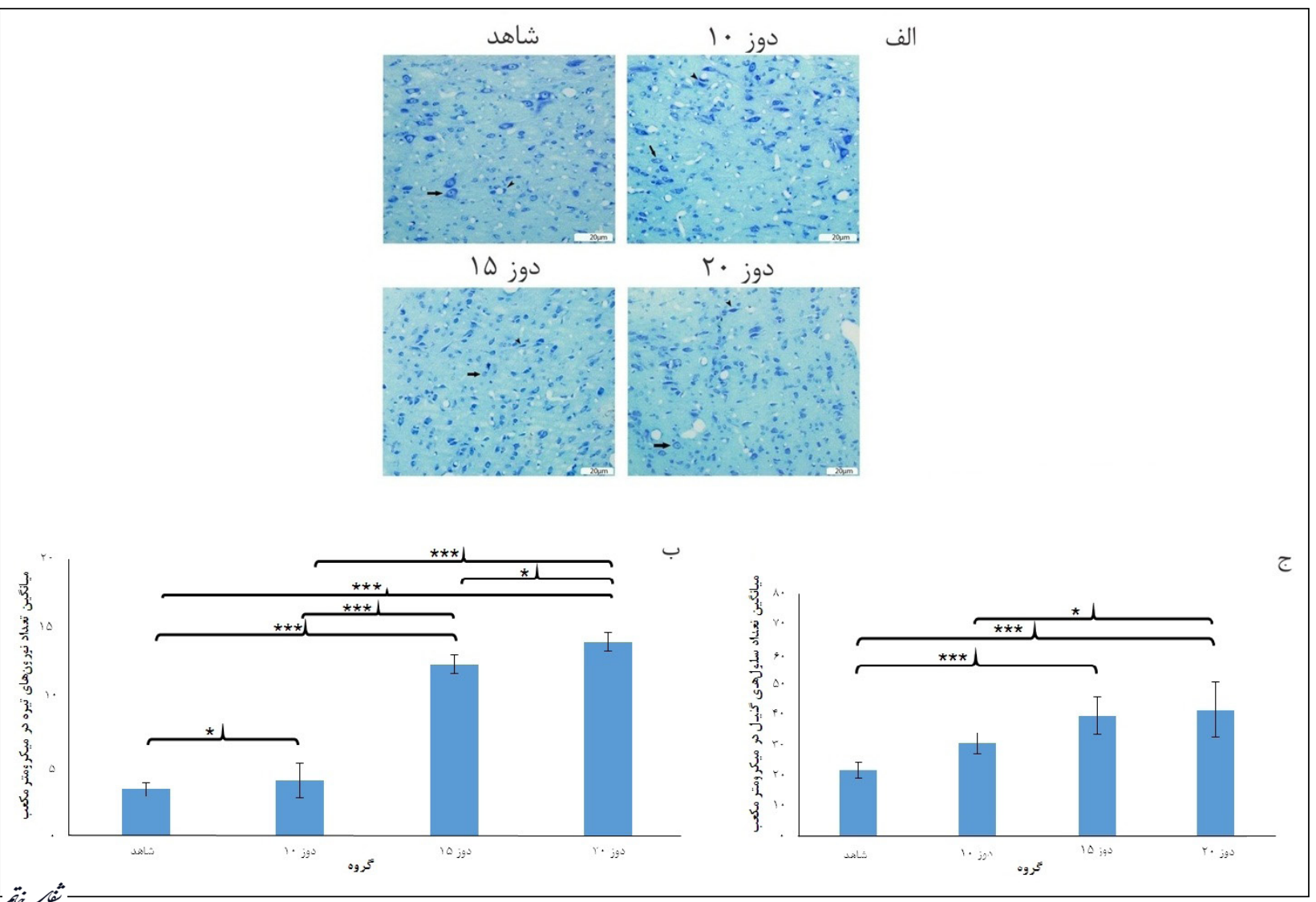

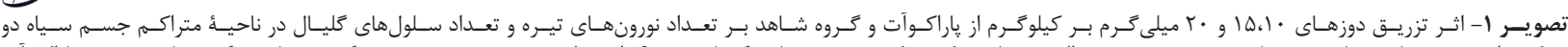

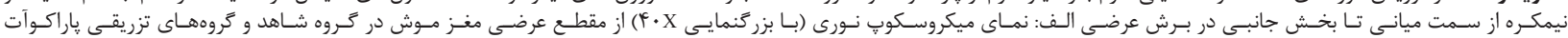

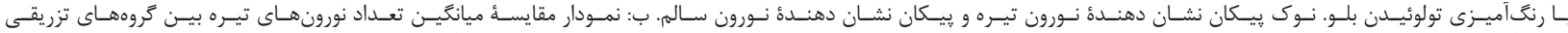

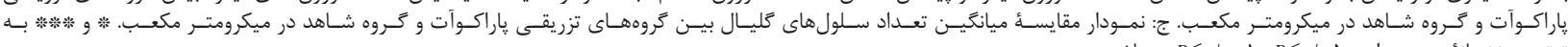

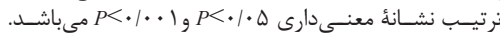

${ }^{18}$ One way ANOVA

${ }^{19}$ Tukey 


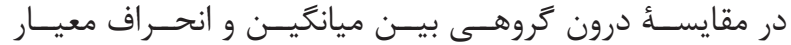

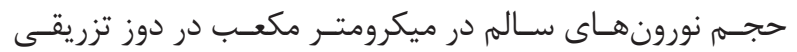

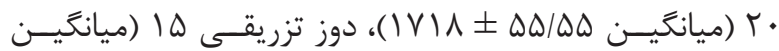

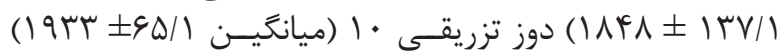
و و Fـ

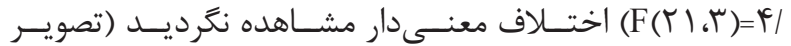

$\cdot\left(3^{-r}\right.$

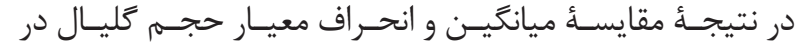

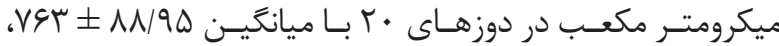

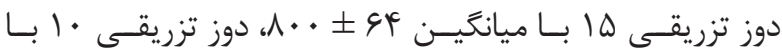

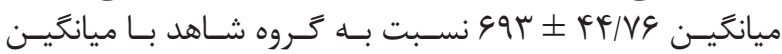

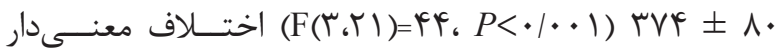

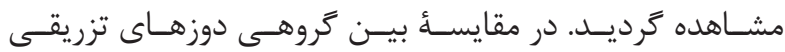

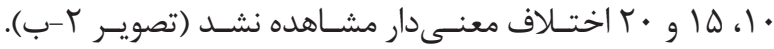

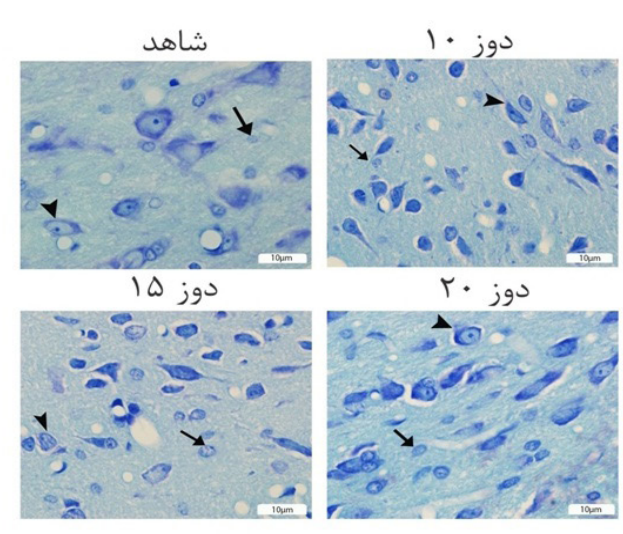

الف
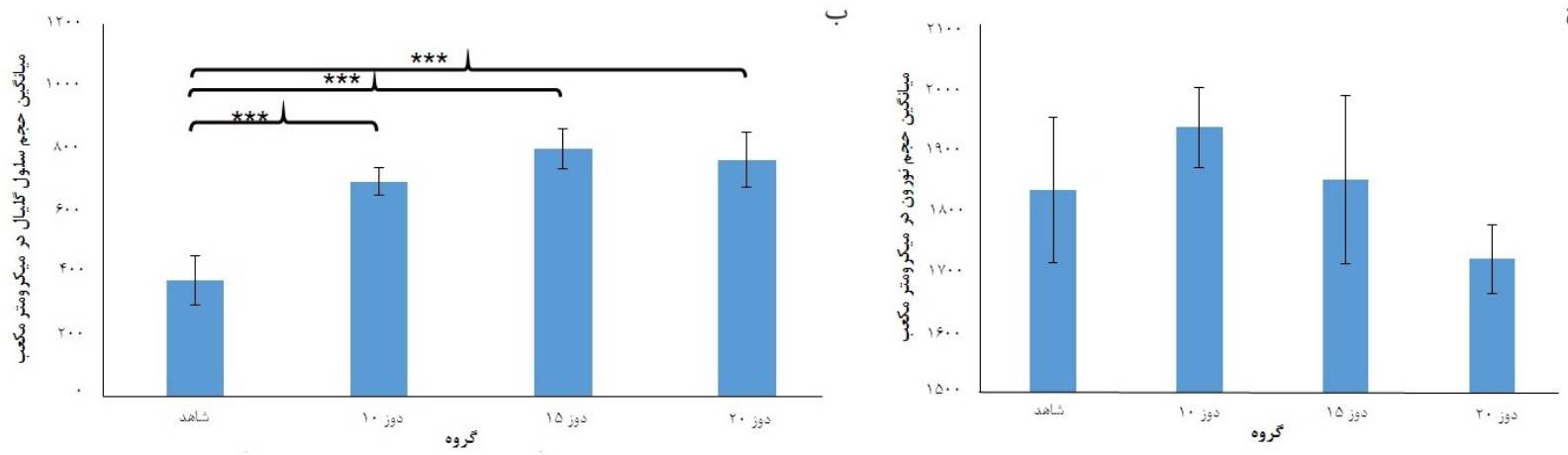

ج

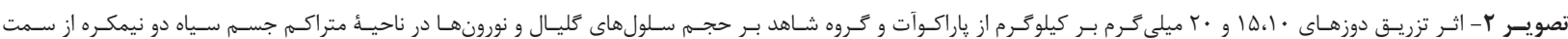

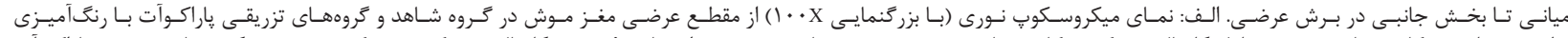

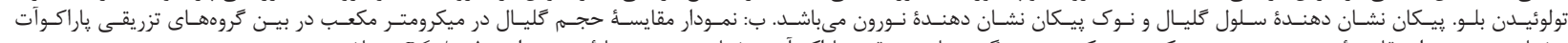

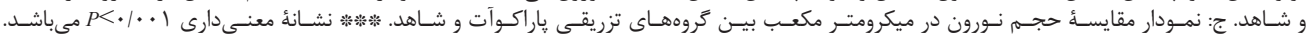

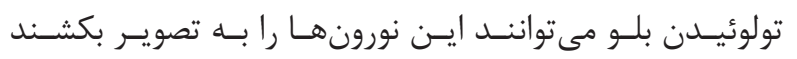

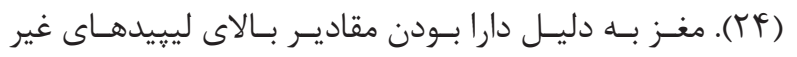

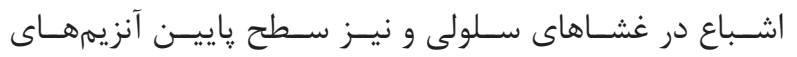

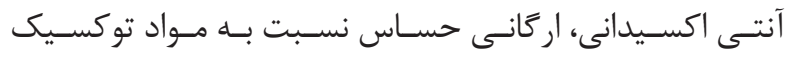
مىباشــد (TQ)

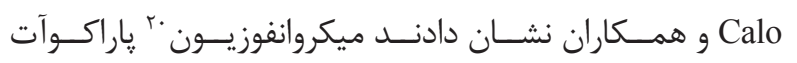

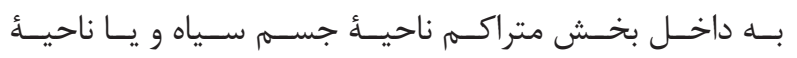

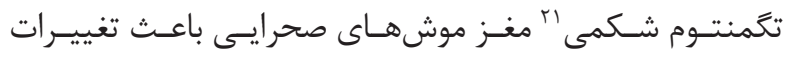

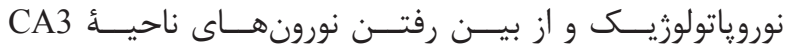

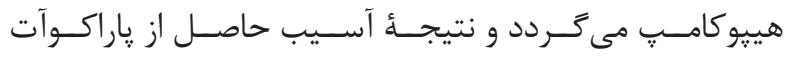

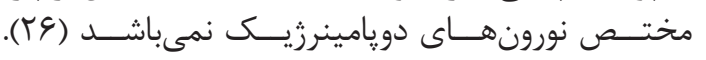

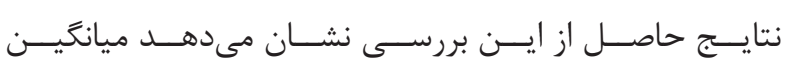

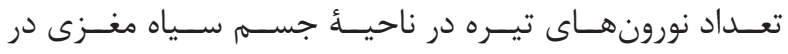

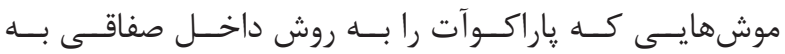

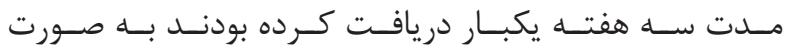

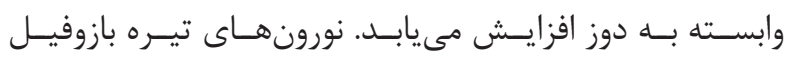

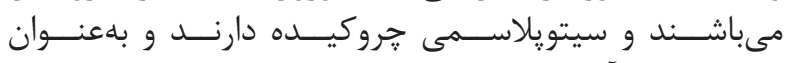

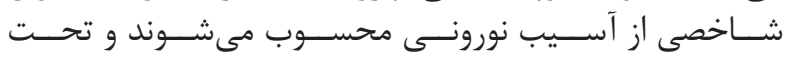

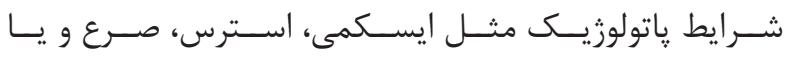

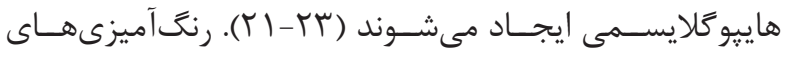

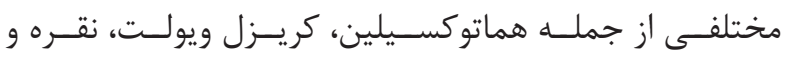




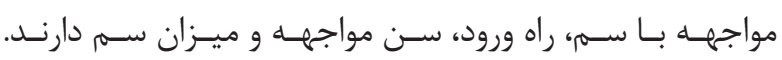

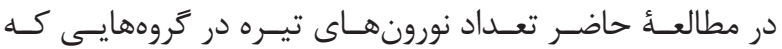

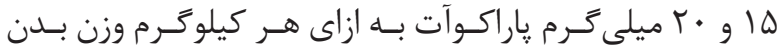

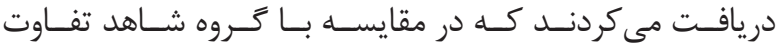

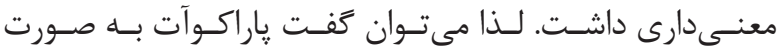

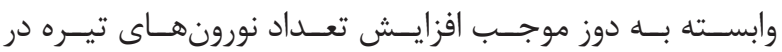

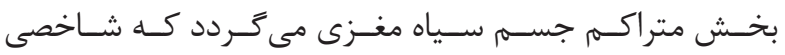

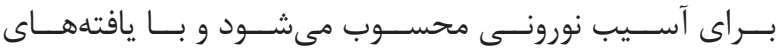
Mc Cormak

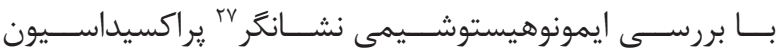

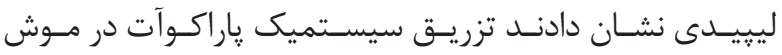

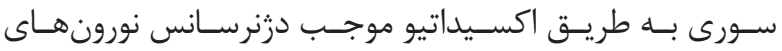

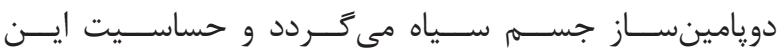

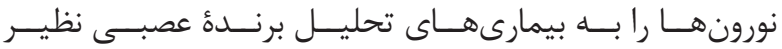

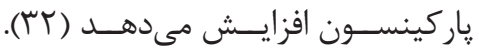

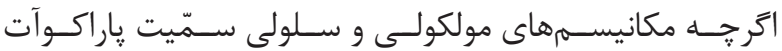

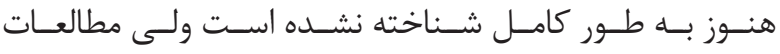

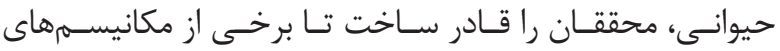

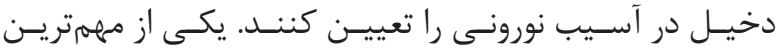

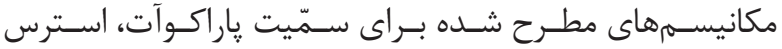

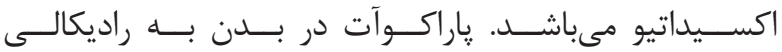

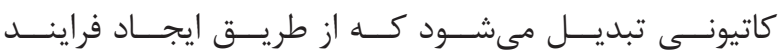

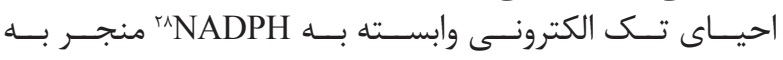

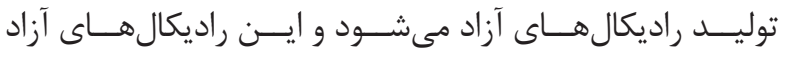

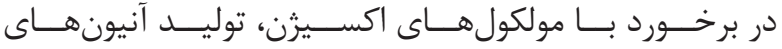

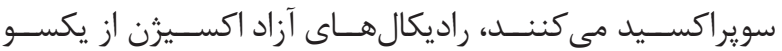

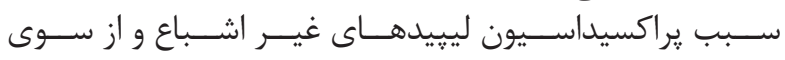

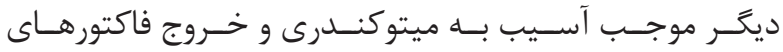

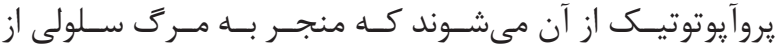

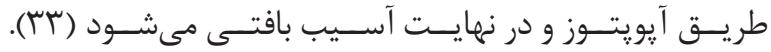

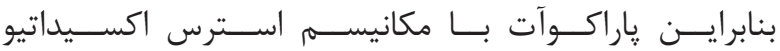

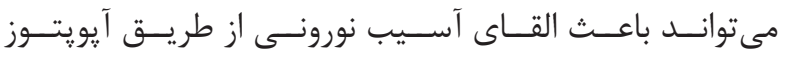

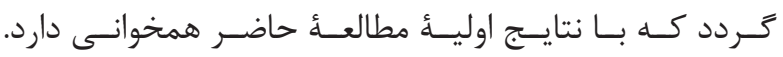

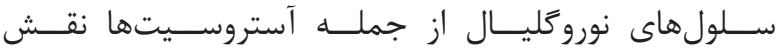

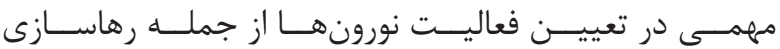

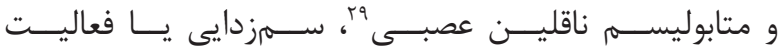

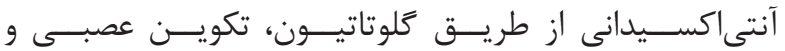

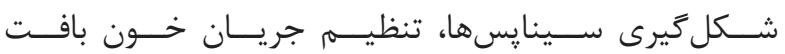

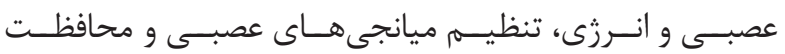

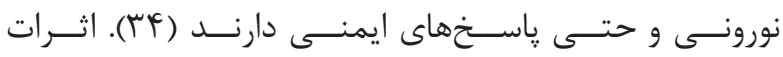

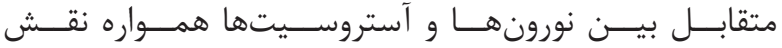

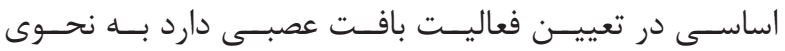

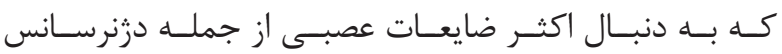

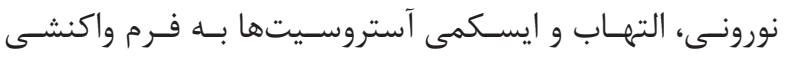

${ }^{22}$ Apomorphine

${ }^{23}$ Amino acid transporters

${ }^{24}$ Tyrosine hydroxylase

${ }^{25}$ Caudate putamen

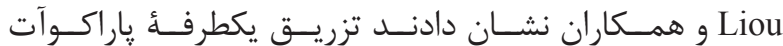

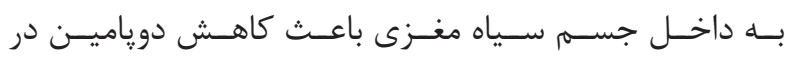

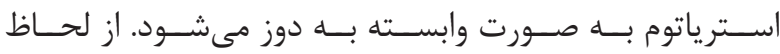

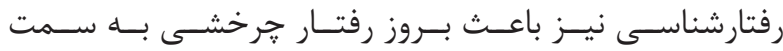

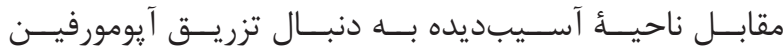

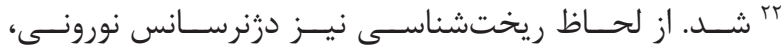

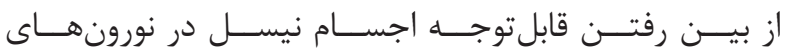

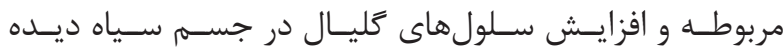

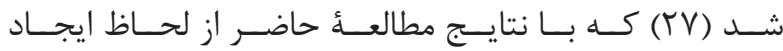

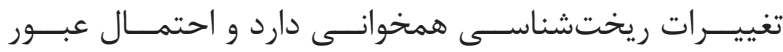

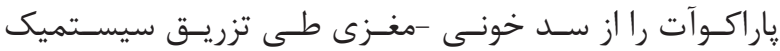

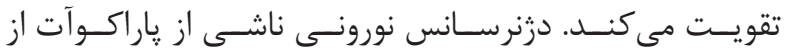

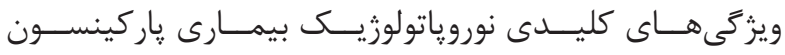

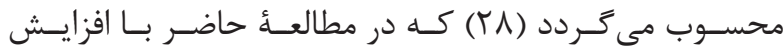

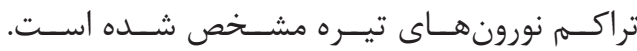

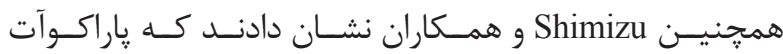

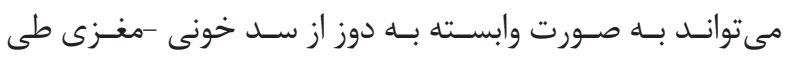

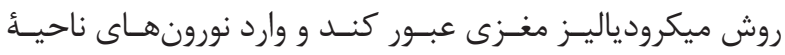

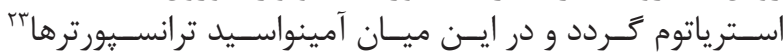

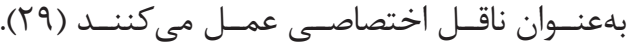

Ossowska

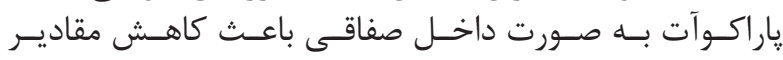

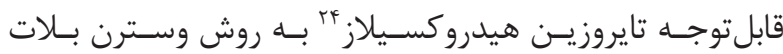

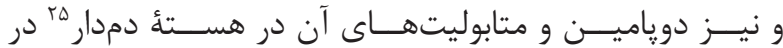

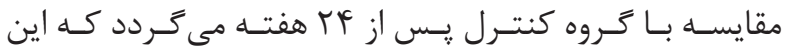

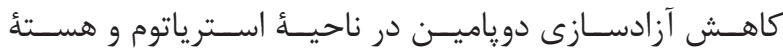

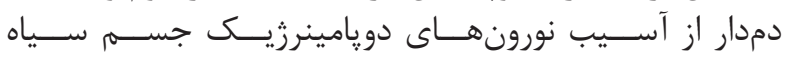

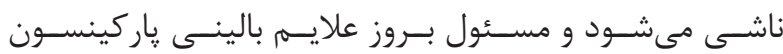

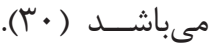

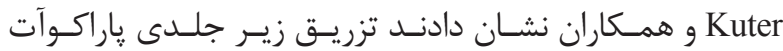

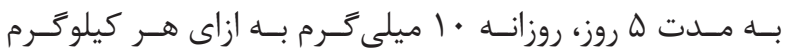

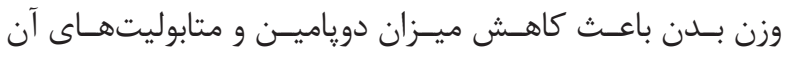

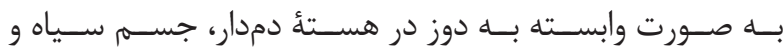

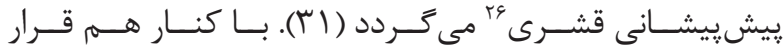

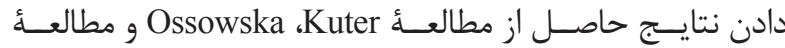

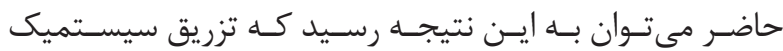

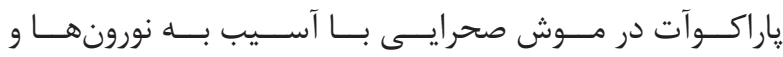

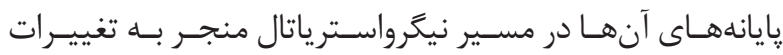

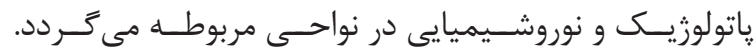

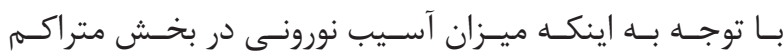

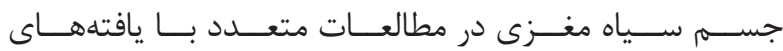

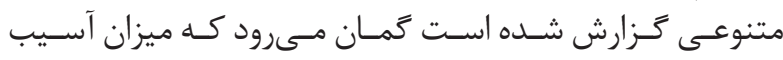

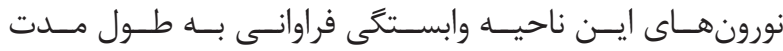

${ }^{26}$ Prefrontal cortex

${ }^{27}$ Marker

${ }^{28}$ Nicotinamide adenine dinucleotide phosphate

${ }^{29}$ Neurotransmitter 


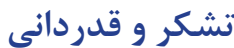

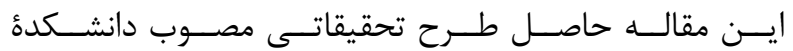

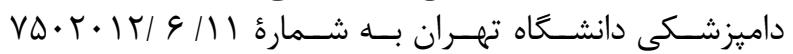

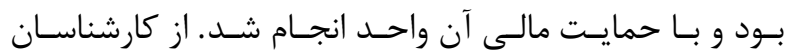

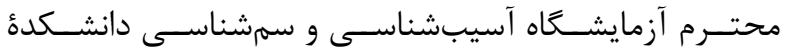

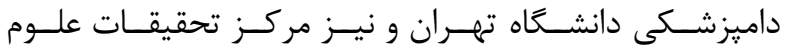

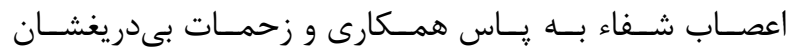

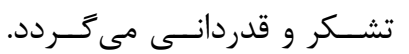

1. Wilks MF, Fernando R, Ariyananda PL, Eddleston M, Berry DJ, Tomenson JA, et al. Improvement in survival after paraquat ingestion following introduction of a new formulation in Sri Lanka. PLoS Med. 2008; 5(2): e49. doi: 10.1371/journal.pmed.0050049.

2. Bertolote JM, Fleischmann A, Eddleston M, Gunnell D. Deaths from pesticide poisoning: a global response. Br J Psychiatry. 2006; 189: 201-3.

3. Jeyaratnam J. Acute pesticide poisoning: a major global health problem. World Health Stat Q. 1990; 43(3): 139-44.

4. Reigart JR. Recognition and management of pesticide poisonings. DIANE Publishing; 2009. p. 11-7.

5. Roberts TR, Dyson JS, Lane MC. Deactivation of the biological activity of paraquat in the soil environment: a review of long-term environmental fate. J Agric Food Chem. 2002; 50(13): 3623-31.

6. Sittipunt C. Paraquat poisoning. Respir Care. 2005; 50(3): 383-5.

7. Izadi MN, Gheshlaghi F, Sharafi SE. Fatal poisoning cases admitted to the emergency department of poisoning, noor hospital. J Clin Forensic Med. 2003; 9(13): $122-6$

8. Bullivant CM. Accidental poisoning by paraquat: Report of two cases in man. BMJ. 1966; 1(5498): 1272.

9. Trush MA, Mimnaugh EG, Ginsburg E, Gram TE. In vitro stimulation by paraquat of reactive oxygenmediated lipid peroxidation in rat lung microsomes. Toxicol Appl Pharmacol. 1981; 60(2): 279-86.

10. Suntres ZE. Role of antioxidants in paraquat toxicity. Toxicology. 2002; 180(1): 65-77.

11. Richardson JR, Quan Y, Sherer TB, Greenamyre

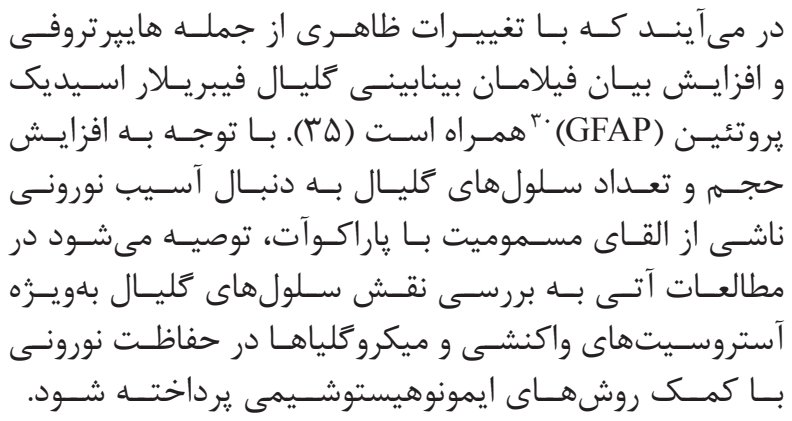

منابع

JT, Miller GW. Paraquat neurotoxicity is distinct from that of MPTP and rotenone. Toxicol Sci. 2005; 88(1): 193-201.

12. Lawrence WS. Geographic variations in the prevalence rates of Parkinson's disease in Alberta. Can J Neurol Sci. 1993; 20(4): 307-11.

13. Ben-Shlomo Y, Finnan F, Allwright S, Davey SG. The epidemiology of Parkinson's disease in the Republic of Ireland: observations from routine data sources. Journal of the Irish Medical Association. 1992; 86(6): 190.1

14. Menegon A, Board PG, Blackburn AC, Mellick GD, Le Couteur DG. Parkinson's disease, pesticides, and glutathione transferase polymorphisms. Lancet. 1998; 352(9137): 1344-6.

15. Gorell JM, Johnson CC, Rybicki BA, Peterson EL, Richardson RJ. The risk of Parkinson's disease with exposure to pesticides, farming, well water, and rural living. Neurol. 1998; 50(5): 1346-50.

16. Gibrat C, Saint-Pierre M, Bousquet M, Lévesque D, Rouillard C, Cicchetti F. Differences between subacute and chronic MPTP mice models: investigation of dopaminergic neuronal degeneration and $\alpha$-synuclein inclusions. J Neurochem. 2009; 109(5): 1469-82.

17. Choi WS, Palmiter RD, Xia Z. Loss of mitochondrial complex I activity potentiates dopamine neuron death induced by microtubule dysfunction in a Parkinson's disease model. J Cell Biol. 2011; 192(5): 873-82.

18. Gazewood JD, Richards DR, Clebak K. Parkinson disease:anupdate.AmFamPhysician.2013;87(4):267-73.

19. Uchenna NK, Nweze KG, Charles II. Effects of the methanolic extract of abeloschus esculentus (L) moench (Okro) fruit on the testes and sperm characteristics of male albino wistar rats. Int J Adv Biol Biom Res. 2014;

${ }^{30}$ Glial fibrillary acidic protein 
2(10): 2686-90.

20. Paxinos G, Watson C. The rat brain in stereotaxic coordinates. San Diego. Academic Press. 1986; p. 15-8.

21. Kherani ZS, Auer RN. Pharmacologic analysis of the mechanism of dark neuron production in cerebral cortex. Acta Neuropathol. 2008; 116(4): 447-52.

22. Auer RN, Kalimo H, Olsson Y, Siesjö BK. The temporal evolution of hypoglycemic brain damage. II. Light- and electron-microscopic findings in the hippocampal gyrus and subiculum of the rat. Acta Neuropathol. 1985; 67(1-2): 13-24.

23. Baracskay P, Szepesi Z, Orbán G, Juhász G, Czurkó A. Generalization of seizures parallels the formation of "dark" neurons in the hippocampus and pontine reticular formation after focal-cortical application of 4-aminopyridine (4-AP) in the rat. Brain Res. 2008; 1228: 217-28.

24. IshidaK, ShimizuH,HidaH,UrakawaS, IdaK, Nishino H. Argyrophilic dark neurons represent various states of neuronal damage in brain insults: some come to die and others survive. Neuroscience. 2004; 125(3): 633-44.

25. Marttila RJ, Röyttä M, Lorentz H, Rinne UK. Oxygen toxicity protecting enzymes in the human brain. J Neural Transm. 1988; 74(2): 87-95.

26. Calò M, Iannone M, Passafaro MM, Nistico G. Selective vulnerability of hippocampal CA3 neurones after microinfusion of paraquat into the rat substantia nigra or into the ventral tegmental area. J Comp Pathol. 1990; 103(1): 73-8.

27. Liou HH, Chen RC, Tsai YF, Chen WP, Chang YC, Tsai MC. Effects of paraquat on the substantia nigra of the Wistar rats: neurochemical, histological, and behavioral studies. Toxicol Appl Pharmacol. 1996; 137(1): 34-41.
28. Purisai MG, McCormack AL, Cumine S, Li J, Isla MZ, Monte DA. Microglial activation as a priming event leading to paraquat-induced dopaminergic cell degeneration. Neurobiol Dis. 2007; 25(2): 392-400.

29. Shimizu K, Ohtaki K, Matsubara K, Aoyama K, Uezono T, Saito O, et al. Carrier-mediated processes in blood-brain barrier penetration and neural uptake of paraquat. Brain Res. 2001; 906(1): 135-42.

30. Ossowska K, Wardas J, Śmiałowska M, Kuter K, Lenda T, Wierońska JM, et al. A slowly developing dysfunction of dopaminergic nigrostriatal neurons induced by long-term paraquat administration in rats: an animal model of preclinical stages of Parkinson's disease? Eur J Neurosci. 2005; 22(6): 1294-304.

31. Kuter K, Śmiałowska M, Wierońska J, Zięba B, Wardas J, Pietraszek M, et al. Toxic influence of subchronic paraquat administration on dopaminergic neurons in rats. Brain Res. 2007; 1155: 196-207.

32. McCormack AL, Thiruchelvam M, Manning-Bog AB, Thiffault C, Langston JW, Cory-Slechta DA, et al. Environmental risk factors and Parkinson's disease: selective degeneration of nigral dopaminergic neurons caused by the herbicide paraquat. Neurobiol. Dis. 2002; 10(2): 119-27.

33. Ramachandiran S, Hansen JM, Jones DP, Richardson JR, Miller GW. Divergent mechanisms of paraquat, $\mathrm{MPP}+$, and rotenone toxicity: oxidation of thioredoxin and caspase-3 activation. Toxicol Sci. 2007; 95(1): 163-71

34. Seth P, Koul N. Astrocyte, the star avatar: redefined. J Biosci. 2008; 33(3): 405-21.

35. Maragakis NJ, Rothstein JD. Mechanisms of disease: astrocytes in neurodegenerative disease. Nat Rev Neurol. 2006; 2(12): 679-89. 